\title{
EQUAL SUMS OF FOUR SEVENTH POWERS
}

\author{
RANDY L. EKL
}

\begin{abstract}
In this paper, the method used to find the smallest, nontrivial, positive integer solution of $a_{1}^{7}+a_{2}^{7}+a_{3}^{7}+a_{4}^{7}=b_{1}^{7}+b_{2}^{7}+b_{3}^{7}+b_{4}^{7}$ is discussed. The solution is

$$
149^{7}+123^{7}+14^{7}+10^{7}=146^{7}+129^{7}+90^{7}+15^{7} .
$$

Factors enabling this discovery are advances in computing power, available workstation memory, and the appropriate choice of optimized algorithms.
\end{abstract}

\section{INTRODUCTION}

Diophantine equations of the form $\sum_{i=1}^{m} a_{i}^{s}=\sum_{j=1}^{n} b_{j}^{s}$ have been studied by mathematicians since antiquity. Classes of this equation are the Pythagorean Theorem $(n=1, m=s=2)$, Fermat's Last Theorem $(n=1, m=2, s>2)$, Euler's Conjecture $(n=1, s>m>2)$, and the Deficient Symmetric Equal Sum of Like Powers $(n>1, m=n, s>m)$.

As reported in [1], [3] and [4], and this article for $[s, m]=[7,4]$, the smallest numeric solutions for several instances of $n>1, m=n, s>m$ are known. Smallest means that $\sum_{i=1}^{m} a_{i}^{s}$ is a minimum.

This paper describes the method used to find the following minimal sum solution for the case $[s, m]=[7,4]$ :

$$
149^{7}+123^{7}+14^{7}+10^{7}=146^{7}+129^{7}+90^{7}+15^{7} .
$$

The second smallest solution is $194^{7}+150^{7}+105^{7}+23^{7}=192^{7}+152^{7}+132^{7}+38^{7}$. The method relies on keeping a sorted, RAM resident, binary tree of values $\sum_{i=1}^{4} a_{i}^{7}$, using a modified AVL tree algorithm (see [2]), precalculating seventh powers of integers, and utilizing large-integer numeric software.

\section{HARDWARE}

The hardware platform used was an HP 715 Unix workstation, with 64 M bytes of RAM, and an HP PRISM architecture RISC processor running at $50 \mathrm{MHz}$. The system had a floating-point coprocessor.

\section{SOFTWARE}

A program written in the "C" language, 514 lines long, was developed. The main data structure used in the program is an AVL tree. It is a height-balanced

Received by the editor May 10, 1995 and, in revised form, July 5, 1995 and September 7, 1995. 1991 Mathematics Subject Classification. Primary 11D41, 11Y50; Secondary 11P05.

Key words and phrases. Diophantine equation, number theory.

(C)1996 American Mathematical Society 
binary tree which contains 4 -tuples $\left(a_{1}, a_{2}, a_{3}, a_{4}\right)$ and their associated values $a_{1}^{7}+$ $a_{2}^{7}+a_{3}^{7}+a_{4}^{7}$.

The initialization section of the program precalculates the seventh powers of integers for use later in the program. Also, in initialization, the AVL tree is seeded with the 4 -tuple $(2,1,1,1)$ and the associated value $2^{7}+1^{7}+1^{7}+1^{7}=131$.

The processing which occurs in the main loop of the program is a methodical generation of 4-tuples $\left(a_{1}, a_{2}, a_{3}, a_{4}\right)$ and their associated sums $a_{1}^{7}+a_{2}^{7}+a_{3}^{7}+a_{4}^{7}$, insertion of them into the AVL tree, and the removal of entries from the AVL tree which can no longer be part of a solution. A call to the avl_remove routine takes the lowest value sum out of the tree and rebalances the tree. The avl_remove routine returns the 4 -tuple $\left(a_{1}, a_{2}, a_{3}, a_{4}\right)$ which produced the sum. Calls to the avl_insert routine are based on 4 -tuples returned by the avl_remove routine. If, upon entering a new value into the AVL tree, it is found that this value already exists in the tree, a solution has been found, as two separate 4-tuples have generated the same sum.

The software was tested on other equations of the form $\sum_{i=1}^{m} a_{i}^{s}=\sum_{j=1}^{m} b_{j}^{s}$, with $m=2,3$ and $s=3,4,5,6$. It found all solutions given in [4]. The search for the case [5,2] was executed up to the sum $2.4 \times 10^{22}$ without finding a solution, and for the case $[6,2]$ was executed up to the sum $7.25 \times 10^{26}$ without finding a solution.

\section{COMPUTATIONAL STATISTICS}

The AVL tree was seeded with $(2,1,1,1)$. The program ran through 20,602,188 iterations (removal calls) before displaying the smallest solution indicated above. The largest that the AVL tree became was 178,345 entries. The program took 3206.41 CPU seconds (53 minutes, 26.41 seconds), or an average of 6425.31 iterations per CPU second. The main need for memory came from two items: 1000 * $6 *$ (long int) $=24 \mathrm{~K}$ bytes was for the power table, and $178,345 * 13 *$ (long int) $=8.9 \mathrm{M}$ bytes was for the AVL tree. The maximum height of the AVL tree was $\log _{2}(178345)+1=18$.

\section{CONCLUSION}

Other noteworthy unsolved problems of the form $\sum_{i=1}^{m} a_{i}^{s}=\sum_{j=1}^{m} b_{j}^{s}$ are $[s, m]=$ $[5,2],[6,2],[7,2]$ and $[7,3]$. It is interesting to observe that in each of these cases, $s>2 m$. Is there a solution to any of these equations? The "next" unsolved case with $m<s \leq 2 m$ is $[s, m]=[8,4]$. The computational methods employed for the problem $[7,4]$ discussed in this paper are applicable to all of the above cases.

\section{REFERENCES}

1. Richard K. Guy, Unsolved Problems in Number Theory, Second Edition, Springer-Verlag, New York, 1994. CMP 95:02

2. Ellis Horowitz and Sartaj Sahni, Fundamentals of Data Structures, Computer Science Press, Inc., Potomac, MD, 1976. MR 54:6540

3. G. H. Hardy and E. M. Wright, Introduction to the Theory of Numbers, 5th edition, Oxford University Press, New York, 1980. MR 81i:10002

4. L. J. Lander, T. R. Parkin and J. L. Selfridge, A Survey of Equal Sums of Like Powers, Mathematics of Computation 21 (1967), 446-459. MR 36:5060

930 Lancaster Lane, Lake Zurich, Illinois 60047

E-mail address: randye@comm.mot.com 